\title{
Optimal Jammer Placement in Wireless Localization Networks
}

\author{
Sinan Gezici*, Suat Bayram ${ }^{b}$, Mohammad Reza Gholami ${ }^{\natural}$, and Magnus Jansson ${ }^{\natural}$ \\ * Department of Electrical and Electronics Engineering, Bilkent University, 06800, Ankara, Turkey \\ $b$ Department of Electrical and Electronics Engineering, Turgut Ozal University, 06010, Ankara, Turkey \\ $\natural$ ACCESS Linnaeus Center, Electrical Eng., KTH Royal Institute of Technology, 100 44, Stockholm, Sweden \\ Emails: gezici@ee.bilkent.edu.tr, sbayram@turgutozal.edu.tr,mohrg@kth.se, janssonm@kth.se
}

\begin{abstract}
The optimal jammer placement problem is proposed for a wireless localization network, where the aim is to degrade the accuracy of locating target nodes as much as possible. In particular, the optimal location of a jammer node is obtained in order to maximize the minimum of the Cramér-Rao lower bounds for a number of target nodes under location related constraints for the jammer node. Theoretical results are derived to specify scenarios in which the jammer node should be located as close to a certain target node as possible, or the optimal location of the jammer node is determined by two or three of the target nodes. In addition, explicit expressions for the optimal location of the jammer node are derived in the presence of two target nodes. Numerical examples are presented to illustrate the theoretical results.
\end{abstract}

Keywords: Localization, jammer, Cramér-Rao lower bound, max-min.

\section{INTRODUCTION}

Position information has a significant role for many location aware services/applications in current and next generation wireless networks. In the absence of GPS signals, e.g., due to lack of access to GPS satellites in some indoor environments, the position information can be extracted from a network consisting of a number of anchor nodes at known locations via, e.g., time-of-arrival measurements [1]. In such wireless localization networks, the main aim is to achieve high localization accuracy, which is commonly defined in terms of the mean squared position error [2].

Although the topic of wireless localization has been studied intensely in various contexts, the effects of jamming on wireless localization networks have gathered little attention in the literature. Recently, a wireless localization scenario is considered in the presence of jammer nodes, which aim to degrade the localization accuracy of the network [3]. The optimal power allocation strategies are proposed for the jammer nodes in order to maximize the average or the minimum Cramér-Rao lower bounds (CRLBs) of the target nodes. The obtained results are useful for quantifying the effects of jamming in wireless localization systems [3].

\footnotetext{
${ }^{0}$ This work was supported in part by the European Commission in the framework of the FP7 Network of Excellence in Wireless COMmunications NEWCOM \# (contract no. 318306). S. Gezici's research was also supported in part by the Distinguished Young Scientist Award of Turkish Academy of Sciences (TUBA-GEBIP 2013).
}

The study in [3] considers fixed locations for the jammer nodes and aims to perform optimal power allocation. In this paper, the main purpose is to obtain the optimal location of a jammer node in order to achieve the best jamming performance in a wireless localization network consisting of multiple target nodes. In particular, the optimal location of the jammer node is investigated in order to maximize the minimum of the CRLBs for the target nodes in a wireless localization network (subject to certain constraints on the location of the jammer node). Although there exist some studies that investigate the jammer placement problem for communication systems, e.g., to prevent eavesdroppers [4] or to jam wireless mesh networks [5], the optimal jammer placement problem has not been considered before for wireless localization networks, to the best of authors' knowledge.

The main contributions of this paper can be summarized as follows: (i) The optimal jammer placement problem in a wireless localization network is proposed for the first time. (ii) It is shown that the jammer node should be as close to a certain target node as possible in certain cases (Proposition 1). (iii) For the case of two target nodes, the location of the jammer node is specified explicitly (Proposition 3). (iv) It is obtained that the optimal jammer location is determined by two or three of the target nodes in certain scenarios (Propositions 2 and 4). Simulation results confirm the theoretical findings.

\section{System Model}

Consider a wireless localization network in a twodimensional space consisting of $N_{A}$ anchor nodes and $N_{T}$ target nodes located at $\boldsymbol{y}_{i} \in \mathbb{R}^{2}, i=1, \ldots, N_{A}$ and $\boldsymbol{x}_{i} \in \mathbb{R}^{2}, i=1, \ldots, N_{T}$, respectively. The target nodes are assumed to estimate their locations based on received signals from the anchor nodes, which have known locations; i.e., self-positioning is considered [2]. ${ }^{1}$ In addition to the target and anchor nodes, there exist a jammer node at $z \in \mathbb{R}^{2}$, which aims to degrade the localization performance of the network. The jammer node is assumed to transmit zero-mean Gaussian noise [5]-[8]. An example of the proposed network model is

\footnotetext{
${ }^{1}$ The problem formulation in this study can be extended to scenarios with remote (network centric) positioning, in which the anchors estimate the locations of the targets.
} 
shown in Fig. 1, with four anchor nodes $\left(N_{A}=4\right)$, four target nodes $\left(N_{T}=4\right)$, and a jammer node.

In this paper, non-cooperative localization is studied, where target nodes receive signals only from anchor nodes (i.e., not from other target nodes) for localization purposes. Also, the connectivity sets are defined as $\mathcal{A}_{i} \triangleq\{j \in$ $\left\{1, \ldots, N_{A}\right\} \mid$ anchor node $j$ is connected to target node $i$ for $i \in\left\{1, \ldots, N_{T}\right\}$. Then, the received signal at target node $i$ coming from anchor node $j$ is expressed as [3]

$$
r_{i j}(t)=\sum_{k=1}^{L_{i j}} \alpha_{i j}^{k} s\left(t-\tau_{i j}^{k}\right)+\gamma_{i} \sqrt{P_{J}} v_{i}(t)+n_{i j}(t)
$$

for $t \in\left[0, T_{\text {obs }}\right], i \in\left\{1, \ldots, N_{T}\right\}$ and $j \in \mathcal{A}_{i}$, where $T_{\mathrm{obs}}$ is the observation time, $\alpha_{i j}^{k}$ and $\tau_{i j}^{k}$ represent, respectively, the amplitude and delay of the $k$ th multipath component between anchor node $j$ and target node $i$, $L_{i j}$ is the number of paths between target node $i$ and anchor node $j$, and $\gamma_{i}$ denotes the channel coefficient between target node $i$ and the jammer node, which has a transmit power of $P_{J}$. The transmit signal $s(t)$ is assumed to be known, and the measurement noise $n_{i j}(t)$ and the jammer noise $\sqrt{P_{J}} v_{i}(t)$ are modeled to be independent zero-mean white Gaussian random processes, where the average power of $n_{i j}(t)$ is $N_{0} / 2$ and that of $v_{i}(t)$ is equal to one [3]. The delay $\tau_{i j}^{k}$ is expressed as

$$
\tau_{i j}^{k} \triangleq \frac{\left\|\boldsymbol{y}_{j}-\boldsymbol{x}_{i}\right\|+b_{i j}^{k}}{c}
$$

with $b_{i j}^{k} \geq 0$ representing a range bias and $c$ being the speed of propagation. Set $\mathcal{A}_{i}$ is partitioned as follows:

$$
\mathcal{A}_{i} \triangleq \mathcal{A}_{i}^{L} \cup \mathcal{A}_{i}^{N L}
$$

where $\mathcal{A}_{i}^{L}$ and $\mathcal{A}_{i}^{N L}$ denote the sets of anchors nodes with line-of-sight (LOS) and non-line-of-sight (NLOS) connections to target node $i$, respectively.

\section{CRLB FOR LOCATION ESTIMATION OF TARGET Nodes}

Regarding target node $i$, the following vector consisting of the bias terms in the LOS and NLOS cases is defined:

$$
\boldsymbol{b}_{i j}= \begin{cases}{\left[b_{i j}^{2} \ldots b_{i j}^{L_{i j}}\right]^{T},} & \text { if } j \in \mathcal{A}_{i}^{L} \\ {\left[b_{i j}^{1} \ldots b_{i j}^{L_{i j}}\right]^{T},} & \text { if } j \in \mathcal{A}_{i}^{N L}\end{cases}
$$

From (4), the unknown parameters related to target node $i$ are defined as follows [9]:

$$
\boldsymbol{\theta}_{i} \triangleq\left[\begin{array}{llll}
\boldsymbol{x}_{i}^{T} & \boldsymbol{b}_{i \mathcal{A}_{i}(1)}^{T} & \cdots & \boldsymbol{b}_{i \mathcal{A}_{i}\left(\left|\mathcal{A}_{i}\right|\right)}^{T}
\end{array}\right]^{T},
$$

where $\mathcal{A}_{i}(j)$ represents the $j$ th element of set $\mathcal{A}_{i}$ and $\left|\mathcal{A}_{i}\right|$ is the number of elements in $\mathcal{A}_{i}$.

The CRLB for location estimation is expressed as [9]

$$
\mathbb{E}\left\{\left\|\hat{\boldsymbol{x}}_{i}-\boldsymbol{x}_{i}\right\|^{2}\right\} \geq \operatorname{tr}\left\{\left[\boldsymbol{F}_{i}^{-1}\right]_{2 \times 2}\right\},
$$

where $\hat{\boldsymbol{x}}_{i}$ represents an unbiased estimate of the location of target node $i, \operatorname{tr}$ denotes the trace operator, and $\boldsymbol{F}_{i}$ is the Fisher information matrix for vector $\boldsymbol{\theta}_{i}$. Based on the steps in [10], $\left[\boldsymbol{F}_{i}^{-1}\right]_{2 \times 2}$ in (6) can be stated as

$$
\left[\boldsymbol{F}_{i}^{-1}\right]_{2 \times 2}=\boldsymbol{J}_{i}\left(\boldsymbol{x}_{i}, P_{J}\right)^{-1}
$$

where the equivalent Fisher information matrix $\boldsymbol{J}_{i}\left(\boldsymbol{x}_{i}, P_{J}\right)$ in the absence of prior information about the location of the target node is expressed as

$$
\boldsymbol{J}_{i}\left(\boldsymbol{x}_{i}, P_{J}\right)=\sum_{j \in \mathcal{A}_{i}^{L}} \frac{\lambda_{i j}}{N_{0} / 2+P_{J}\left|\gamma_{i}\right|^{2}} \boldsymbol{\phi}_{i j} \boldsymbol{\phi}_{i j}^{T}
$$

with

$$
\begin{aligned}
\lambda_{i j} & \triangleq \frac{4 \pi^{2} \beta^{2}\left|\alpha_{i j}^{1}\right|^{2} \int_{-\infty}^{\infty}|S(f)|^{2} d f}{c^{2}}\left(1-\xi_{j}\right), \\
\phi_{i j} & \triangleq\left[\begin{array}{ll}
\cos \varphi_{i j} & \sin \varphi_{i j}
\end{array}\right]^{T} .
\end{aligned}
$$

In (9), $\beta$ denotes the effective bandwidth, and is given by

$$
\beta=\sqrt{\frac{\int_{-\infty}^{\infty} f^{2}|S(f)|^{2} d f}{\int_{-\infty}^{\infty}|S(f)|^{2} d f}},
$$

with $S(f)$ representing the Fourier transform of $s(t)$, and the path-overlap coefficient $\xi_{j}$ is a non-negative number between zero and one, that is, $0 \leq \xi_{j} \leq 1$ [11]. In addition, $\varphi_{i j}$ in (10) denotes the angle between target node $i$ and anchor node $j$.

From (6)-(8), the CRLB for target node $i$ can be obtained as follows:

$$
\mathrm{CRLB}_{i}=\operatorname{tr}\left\{\boldsymbol{J}_{i}\left(\boldsymbol{x}_{i}, P_{J}\right)^{-1}\right\}=r_{i}\left(P_{J}\left|\gamma_{i}\right|^{2}+N_{0} / 2\right)
$$

where

$$
r_{i} \triangleq \operatorname{tr}\left\{\left[\sum_{j \in \mathcal{A}_{i}^{L}} \lambda_{i j} \phi_{i j} \phi_{i j}^{T}\right]^{-1}\right\} .
$$

\section{Optimal Jammer Placement}

\section{A. Generic Formulation and Analysis}

The aim is to determine the optimal position for the jammer node in order to increase the CRLBs of all the target nodes as much as possible. The CRLB is considered as a performance metric since it bounds the localization performance of a target node in terms of the mean-squared error [12]-[14]. In particular, the minimum of the CRLBs of the target nodes is considered as the objective function to guarantee that all the target nodes have localization accuracy bounded by a certain limit. The proposed problem formulation is expressed, based on (12), as follows:

$$
\begin{aligned}
& \underset{\boldsymbol{z}}{\operatorname{maximize}} \min _{i \in\left\{1, \ldots, N_{T}\right\}} r_{i}\left(P_{J}\left|\gamma_{i}\right|^{2}+\frac{N_{0}}{2}\right) \\
& \text { subject to }\left\|\boldsymbol{z}-\boldsymbol{x}_{i}\right\| \geq \varepsilon, \quad i=1, \ldots, N_{T}
\end{aligned}
$$

where $\varepsilon>0$ denotes the lower limit for the distance between a target node and the jammer node, which is 
incorporated into the formulation since it may not be possible for the jammer node to get very close to target nodes in practical jamming scenarios (e.g., the jammer node may need to hide) [5].

Similarly to [12] and [15], the channel power gain between the jammer node and the $i$ th target node is modeled as

$$
\left|\gamma_{i}\right|^{2}=\tilde{K}_{i}\left(\frac{d_{0}}{\left\|\boldsymbol{z}-\boldsymbol{x}_{i}\right\|}\right)^{\nu}
$$

for $\left\|\boldsymbol{z}-\boldsymbol{x}_{i}\right\|>d_{0}$, where $d_{0}$ is the reference distance for the antenna far-field, $\nu$ is the path-loss exponent (commonly between 2 and 4 ), and $\tilde{K}_{i}$ is a unitless constant that depends on antenna characteristics and average channel attenuation [16]. It is assumed that $\tilde{K}_{i}$ 's, $d_{0}, \nu$, and $\varepsilon$ are known, and that $\varepsilon>d_{0}$. From (15), the optimization problem in (14) can be expressed as follows: ${ }^{2}$

$$
\begin{gathered}
\underset{\boldsymbol{z}}{\operatorname{maximize}} \min _{i \in\left\{1, \ldots, N_{T}\right\}} r_{i}\left(\frac{K_{i} P_{J}}{\left\|\boldsymbol{z}-\boldsymbol{x}_{i}\right\|^{\nu}}+\frac{N_{0}}{2}\right) \\
\text { subject to }\left\|\boldsymbol{z}-\boldsymbol{x}_{i}\right\| \geq \varepsilon, \quad i=1, \ldots, N_{T}
\end{gathered}
$$

where $K_{i} \triangleq \tilde{K}_{i}\left(d_{0}\right)^{\nu}$. The problem in (16) is non-convex; hence, convex optimization tools cannot be employed to obtain the optimal location of the jammer node. Therefore, an exhaustive search over the feasible locations for the jammer node may be required in general. However, some theoretical results are obtained in the following in order to simplify the optimization problem in (16) under various conditions.

Proposition 1: If there exists a target node, say the lth one, that satisfies the following inequality,

$$
\begin{aligned}
& r_{\ell}\left(\frac{K_{\ell} P_{J}}{\varepsilon^{\nu}}+\frac{N_{0}}{2}\right) \\
& \quad \leq \min _{\substack{i \in\left\{1, \ldots, N_{T}\right\} \\
i \neq \ell}} r_{i}\left(\frac{K_{i} P_{J}}{\left(\left\|\boldsymbol{x}_{i}-\boldsymbol{x}_{\ell}\right\|+\varepsilon\right)^{\nu}}+\frac{N_{0}}{2}\right)
\end{aligned}
$$

and if set $\left\{\boldsymbol{z}:\left\|\boldsymbol{z}-\boldsymbol{x}_{\ell}\right\|=\varepsilon \&\left\|\boldsymbol{z}-\boldsymbol{x}_{i}\right\| \geq \varepsilon, i=\right.$ $\left.1, \ldots, \ell-1, \ell+1, \ldots, N_{T}\right\}$ is non-empty, then the solution of (16), denoted by $\boldsymbol{z}^{\mathrm{opt}}$, satisfies $\left\|\boldsymbol{z}^{\mathrm{opt}}-\boldsymbol{x}_{\ell}\right\|=\varepsilon$; that $i s$, the jammer node is placed at a distance of $\varepsilon$ from the eth target node.

Proof: First, an upper bound is derived for the optimization problem in (16) as follows:

$$
\max _{\boldsymbol{z}} \min _{i \in\left\{1, \ldots, N_{T}\right\}} r_{i}\left(\frac{K_{i} P_{J}}{\left\|\boldsymbol{z}-\boldsymbol{x}_{i}\right\|^{\nu}}+\frac{N_{0}}{2}\right)
$$

\footnotetext{
${ }^{2}$ The jammer node is assumed to know the localization related parameters such that it can solve the optimization problem in (16). In practical scenarios, this information may not completely be available to the jammer node. This assumption is made for two purposes: (i) to obtain initial results which can form a basis for further studies on the problem of optimal jammer placement in localization networks, (ii) to derive theoretical limits on the best achievable performance of the jammer node (if the jammer node can learn all the related parameters, the localization accuracy provided in this paper is achieved; otherwise, the localization accuracy is bounded by the provided results [3]).
}

$$
\begin{aligned}
& \leq \max _{\boldsymbol{z}} r_{\ell}\left(\frac{K_{\ell} P_{J}}{\left\|\boldsymbol{z}-\boldsymbol{x}_{\ell}\right\|^{\nu}}+\frac{N_{0}}{2}\right) \\
& =r_{\ell}\left(\frac{K_{\ell} P_{J}}{\varepsilon^{\nu}}+\frac{N_{0}}{2}\right)
\end{aligned}
$$

where the inequality in (19) is by definition, and the equality in (20) is obtained from the constraint in (16). Next, towards the aim of proving the achievability of the upper bound in (20) under the conditions in the proposition, the following relation is presented for $i \in$ $\left\{1, \ldots, N_{T}\right\} \backslash\{\ell\}$ and for all $\boldsymbol{z}$ such that $\left\|\boldsymbol{z}-\boldsymbol{x}_{\ell}\right\|=\varepsilon$ :

$$
\begin{aligned}
r_{i}\left(\frac{K_{i} P_{J}}{\left\|\boldsymbol{z}-\boldsymbol{x}_{i}\right\|^{\nu}}+\frac{N_{0}}{2}\right) & \geq r_{i}\left(\frac{K_{i} P_{J}}{\left(\left\|\boldsymbol{x}_{i}-\boldsymbol{x}_{\ell}\right\|+\varepsilon\right)^{\nu}}+\frac{N_{0}}{2}\right) \\
& \geq r_{\ell}\left(\frac{K_{\ell} P_{J}}{\varepsilon^{\nu}}+\frac{N_{0}}{2}\right)
\end{aligned}
$$

where the first inequality follows from the triangle inequality; that is, $\left\|\boldsymbol{z}-\boldsymbol{x}_{i}\right\| \leq\left\|\boldsymbol{x}_{i}-\boldsymbol{x}_{\ell}\right\|+\left\|\boldsymbol{z}-\boldsymbol{x}_{\ell}\right\|=\| \boldsymbol{x}_{i}-$ $\boldsymbol{x}_{\ell} \|+\varepsilon$, and the second inequality is due to the condition in (17). The inequality in (21) for $i \in\left\{1, \ldots, N_{T}\right\} \backslash\{\ell\}$ implies that, for $\left\|\boldsymbol{z}-\boldsymbol{x}_{\ell}\right\|=\varepsilon$ and under the condition in (17), the upper bound in (20) can be achieved as follows:

$$
\begin{aligned}
& \min _{i \in\left\{1, \ldots, N_{T}\right\}} r_{i}\left(\frac{K_{i} P_{J}}{\left\|\boldsymbol{z}-\boldsymbol{x}_{i}\right\|^{\nu}}+\frac{N_{0}}{2}\right) \\
& =r_{\ell}\left(\frac{K_{\ell} P_{J}}{\left\|\boldsymbol{z}-\boldsymbol{x}_{l}\right\|^{\nu}}+\frac{N_{0}}{2}\right)=r_{\ell}\left(\frac{K_{\ell} P_{J}}{\varepsilon^{\nu}}+\frac{N_{0}}{2}\right)
\end{aligned}
$$

if set $\left\{\boldsymbol{z}:\left\|\boldsymbol{z}-\boldsymbol{x}_{\ell}\right\|=\varepsilon \&\left\|\boldsymbol{z}-\boldsymbol{x}_{i}\right\| \geq \varepsilon, i=1, \ldots, \ell-\right.$ $\left.1, \ell+1, \ldots, N_{T}\right\}$ is non-empty. In other words, under the conditions in the proposition, the optimization problem in (16) achieves the upper bound in (20) for $\left\|\boldsymbol{z}-\boldsymbol{x}_{\ell}\right\|=\varepsilon$. Hence, the solution $\boldsymbol{z}^{\text {opt }}$ of (16) satisfies $\left\|\boldsymbol{z}^{\text {opt }}-\boldsymbol{x}_{\ell}\right\|=\varepsilon$ if the conditions in the proposition hold.

Proposition 1 presents a scenario in which the jammer node must be as close to a certain target node (denoted by target node $\ell$ in the proposition) as possible in order to maximize the minimum of the CRLBs of the target nodes. In that scenario, the feasible set for the jammer location is significantly reduced, which simplifies the search space for the optimization problem in (16).

In order to specify another scenario in which the solution of (16) can be obtained in a simplified manner, consider the optimization problem in (16) in the presence of two target nodes $\ell_{1}$ and $\ell_{2}$ only; that is,

$$
\begin{gathered}
\underset{\boldsymbol{z}}{\operatorname{maximize}} \min _{i \in\left\{\ell_{1}, \ell_{2}\right\}} r_{i}\left(\frac{K_{i} P_{J}}{\left\|\boldsymbol{z}-\boldsymbol{x}_{i}\right\|^{\nu}}+\frac{N_{0}}{2}\right) \\
\text { subject to }\left\|\boldsymbol{z}-\boldsymbol{x}_{\ell_{1}}\right\| \geq \varepsilon,\left\|\boldsymbol{z}-\boldsymbol{x}_{\ell_{2}}\right\| \geq \varepsilon
\end{gathered}
$$

where $\ell_{1}, \ell_{2} \in\left\{1, \ldots, N_{T}\right\}$ and $\ell_{1} \neq \ell_{2}$. Let $\boldsymbol{z}_{\ell_{1}, \ell_{2}}^{\text {opt }}$ and $\mathrm{CRLB}_{\ell_{1}, \ell_{2}}$ denote the optimizer and the optimal value of (24), respectively. (In the next section, the solution in the presence of two target nodes is investigated in detail.) Then, the following proposition characterizes the solution of (16) under certain conditions.

Proposition 2: Let $\mathrm{CRLB}_{k, i}$ be the minimum of $\mathrm{CRLB}_{\ell_{1}, \ell_{2}}$ for $\ell_{1}, \ell_{2} \in\left\{1, \ldots, N_{T}\right\}$ and $\ell_{1} \neq \ell_{2}$, and 
let $\boldsymbol{z}_{k, i}^{\mathrm{opt}}$ denote the corresponding jammer location (i.e., the optimizer of (24) for $\ell_{1}=k$ and $\ell_{2}=i$ ). Then, an optimal jammer location obtained from (16) is equal to $\boldsymbol{z}_{k, i}^{\mathrm{opt}}$ if $\boldsymbol{z}_{k, i}^{\mathrm{opt}}$ is an element of set $\left\{\boldsymbol{z}:\left\|\boldsymbol{z}-\boldsymbol{x}_{m}\right\| \geq\right.$ $\left.\varepsilon, m \in\left\{1, \ldots, N_{T}\right\} \backslash\{k, i\}\right\}$ and

$$
r_{m}\left(\frac{K_{m} P_{J}}{\left\|\boldsymbol{z}_{k, i}^{\mathrm{opt}}-\boldsymbol{x}_{m}\right\|^{\nu}}+\frac{N_{0}}{2}\right) \geq \mathrm{CRLB}_{k, i}
$$

for $m \in\left\{1, \ldots, N_{T}\right\} \backslash\{k, i\}$.

Proposition 2 specifies a scenario in which the optimal jammer location is mainly determined by two of the target nodes since the others have larger CRLBs when the jammer node is placed at the optimal location according to those two jammer nodes only. In such a scenario, the optimal jammer location can be found easily, as the solution of (24) is simple to obtain (in comparison to (16)), which is investigated in the following section.

\section{B. Special Case: Two Target Nodes}

In the case of two target nodes, the solution of (16) can easily be obtained based on the following result.

Proposition 3: For the case of two target nodes (i.e., $N_{T}=2$ ), the solution $z^{\text {opt }}$ of (16) satisfies one of the following conditions:

(i) if $\left\|\boldsymbol{x}_{1}-\boldsymbol{x}_{2}\right\|<2 \varepsilon$, then $\left\|\boldsymbol{z}^{\text {opt }}-\boldsymbol{x}_{1}\right\|=\| \boldsymbol{z}^{\text {opt }}-$ $\boldsymbol{x}_{2} \|=\varepsilon$.

(ii) otherwise,

(a) if $r_{1}\left(\frac{K_{1} P_{J}}{\varepsilon^{\nu}}+\frac{N_{0}}{2}\right) \leq r_{2}\left(\frac{K_{2} P_{J}}{\left(\left\|\boldsymbol{x}_{1}-\boldsymbol{x}_{2}\right\|-\varepsilon\right)^{\nu}}+\frac{N_{0}}{2}\right)$, then $\left\|\boldsymbol{z}^{\mathrm{opt}}-\boldsymbol{x}_{1}\right\|=\varepsilon$ and $\left\|\boldsymbol{z}^{\mathrm{opt}}-\boldsymbol{x}_{2}\right\|=\left\|\boldsymbol{x}_{1}-\boldsymbol{x}_{2}\right\|-\varepsilon$.

(b) if $r_{2}\left(\frac{K_{2} P_{J}}{\varepsilon^{\nu}}+\frac{N_{0}}{2}\right) \leq r_{1}\left(\frac{K_{1} P_{J}}{\left(\left\|\boldsymbol{x}_{1}-\boldsymbol{x}_{2}\right\|-\varepsilon\right)^{\nu}}+\frac{N_{0}}{2}\right)$, then $\left\|\boldsymbol{z}^{\mathrm{opt}}-\boldsymbol{x}_{1}\right\|=\left\|\boldsymbol{x}_{1}-\boldsymbol{x}_{2}\right\|-\varepsilon$ and $\left\|\boldsymbol{z}^{\mathrm{opt}}-\boldsymbol{x}_{2}\right\|=\varepsilon$. (c) otherwise, $\left\|\boldsymbol{z}^{\mathrm{opt}}-\boldsymbol{x}_{1}\right\|=d^{*}$ and $\left\|\boldsymbol{z}^{\mathrm{opt}}-\boldsymbol{x}_{2}\right\|=$ $\left\|\boldsymbol{x}_{1}-\boldsymbol{x}_{2}\right\|-d^{*}$, where $d^{*}$ is the unique solution of the following equation over $d \in\left(\varepsilon,\left\|\boldsymbol{x}_{1}-\boldsymbol{x}_{2}\right\|-\varepsilon\right)$.

$$
r_{1}\left(\frac{K_{1} P_{J}}{d^{\nu}}+\frac{N_{0}}{2}\right)=r_{2}\left(\frac{K_{2} P_{J}}{\left(\left\|\boldsymbol{x}_{1}-\boldsymbol{x}_{2}\right\|-d\right)^{\nu}}+\frac{N_{0}}{2}\right)
$$

Based on Proposition 3, the optimal location of the jammer node can be specified for $N_{T}=2$ as follows: If the distance between the target nodes is smaller than $2 \varepsilon$, then the jammer node is located at one of the two intersections of the circles around the target nodes with radius of $\varepsilon$ each. ${ }^{3}$ Otherwise, the jammer node is always on the straight line that connects the two target nodes; that is, $\left\|\boldsymbol{z}^{\text {opt }}-\boldsymbol{x}_{1}\right\|+\left\|\boldsymbol{z}^{\text {opt }}-\boldsymbol{x}_{2}\right\|=\left\|\boldsymbol{x}_{2}-\boldsymbol{x}_{1}\right\|$. In this case, depending on the CRLB values, the jammer node can be either at a distance of $\varepsilon$ from one of the target nodes (the one with the lower CRLB) or at larger distances than $\varepsilon$ from both of the target nodes. In the first scenario, the optimal jammer position is simply obtained as $\boldsymbol{z}^{\mathrm{opt}}=\boldsymbol{x}_{i}+\left(\boldsymbol{x}_{k}-\boldsymbol{x}_{i}\right) \varepsilon /\left\|\boldsymbol{x}_{k}-\boldsymbol{x}_{i}\right\|$ when the jammer

\footnotetext{
${ }^{3}$ For three-dimensional localization, the jammer node is located on the circle corresponding to the intersection of two spheres around the target nodes with radius of $\varepsilon$ each.
}

node is at a distance of $\varepsilon$ from the $i$ th target node. In the second scenario, an equalizer solution is observed as the CRLBs are equated, and the optimal jammer location is calculated as $\boldsymbol{z}^{\text {opt }}=\boldsymbol{x}_{1}+\left(\boldsymbol{x}_{2}-\boldsymbol{x}_{1}\right) d^{*} /\left\|\boldsymbol{x}_{2}-\boldsymbol{x}_{1}\right\|$, where $d^{*}$ is obtained from (26).

\section{Special Case: Infinitesimally Small $\varepsilon$}

In this scenario, $N_{T} \geq 3$ is considered and the optimal location of the jammer node is obtained in the absence of constraints on the distances between the jammer node and the target nodes; that is, it is assumed that the constraints in (16) are ineffective. Then, the following proposition characterizes the optimal solution for the jammer location.

Proposition 4: Suppose that $N_{T} \geq 3$ and $\varepsilon \rightarrow 0$. Let the max-min CRLB in the presence of target nodes $\ell_{1}, \ell_{2}$, and $\ell_{3}$ only be expressed as $\mathrm{CRLB}_{\ell_{1}, \ell_{2}, \ell_{3}}=$ $\max _{\boldsymbol{z}} \min _{m \in\left\{\ell_{1}, \ell_{2}, \ell_{3}\right\}} r_{m}\left(\frac{K_{m} P_{J}}{\left\|\boldsymbol{z}-\boldsymbol{x}_{m}\right\|^{\nu}}+\frac{N_{0}}{2}\right)$. Also, let target nodes $i, j$, and $k$ achieve the minimum of $\mathrm{CRLB}_{\ell_{1}, \ell_{2}, \ell_{3}}$ for $\ell_{1}, \ell_{2}, \ell_{3} \in\left\{1, \ldots, N_{T}\right\}$ and let $\boldsymbol{z}_{i, j, k}^{\mathrm{opt}}$ denote the jammer location corresponding to $\mathrm{CRLB}_{i, j, k}$. Then, the optimal location for the jammer node (i.e., the optimizer of (16) in the absence of the distance constraints) is equal to $\boldsymbol{z}_{i, j, k}^{\mathrm{opt}}$, and at least two of the CRLBS of the target nodes are equalized by the optimal solution.

The importance of Proposition 4 is related to the statement that the optimal location of the jammer node is determined by no more than three of the target nodes for infinitesimally small $\varepsilon$. Once the optimal location of the jammer node is obtained based on Proposition 4 as $\boldsymbol{z}_{i, j, k}^{\mathrm{opt}}$, if $\boldsymbol{z}_{i, j, k}^{\mathrm{opt}}$ is an element of $\left\{\boldsymbol{z} \mid\left\|\boldsymbol{z}-\boldsymbol{x}_{i}\right\| \geq \varepsilon, i=\right.$ $\left.1, \ldots, N_{T}\right\}$, then it also becomes the solution of (16). Otherwise, (16) yields a different solution.

\section{Numerical EXAmples}

In this section, the propositions in Section IV are illustrated via numerical examples. The parameters in (16) are set as $\varepsilon=1 \mathrm{~m} ., P_{J}=6, N_{0}=2, \nu=2$, and $K_{i}=1$ for $i=1, \ldots, N_{T}$. For each target node, LOS connections to all the anchor nodes are assumed, and $r_{i}$ in (16) is calculated via (13) based on (10) and the following expression: $\lambda_{i j}=100 N_{0}\left\|\boldsymbol{x}_{i}-\boldsymbol{y}_{j}\right\|^{-2} / 2$; that is, the free space propagation model is considered as in [11].

First, a network consisting of four anchor nodes $\left(N_{A}=\right.$ 4 ) and four target nodes $\left(N_{T}=4\right)$ is investigated, where the node locations are as illustrated in Fig. 1. For this scenario, the conditions in Proposition 2 are satisfied for $k=1$ and $i=3$, which means that the solution of the whole network is determined by the subnetwork consisting of target node 1 and target node 3 . Then, based on Proposition 3-(ii)-(c), the optimal location of the jammer node and the corresponding max-min CRLB are calculated as $\boldsymbol{z}_{1,3}^{\mathrm{opt}}=[5.06054 .4697] \mathrm{m}$. and $\mathrm{CRLB}_{1,3}=0.8053 \mathrm{~m}^{2}$, respectively. Since the distances between the target nodes and the optimal location of 


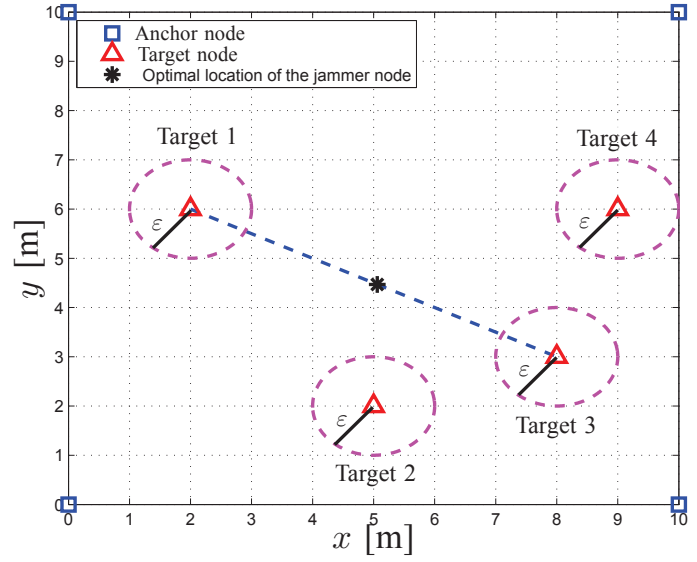

Fig. 1. The network consisting of anchor nodes at [0 0], [10 0], [0 10], and $\left[\begin{array}{ll}10 & 10\end{array}\right] \mathrm{m}$. , and target nodes at $\left[\begin{array}{l}2 \\ 6\end{array}\right],\left[\begin{array}{l}5 \\ 2\end{array}\right],\left[\begin{array}{l}8 \\ 3\end{array}\right]$, and $\left[\begin{array}{ll}9 & 6\end{array}\right] \mathrm{m}$.

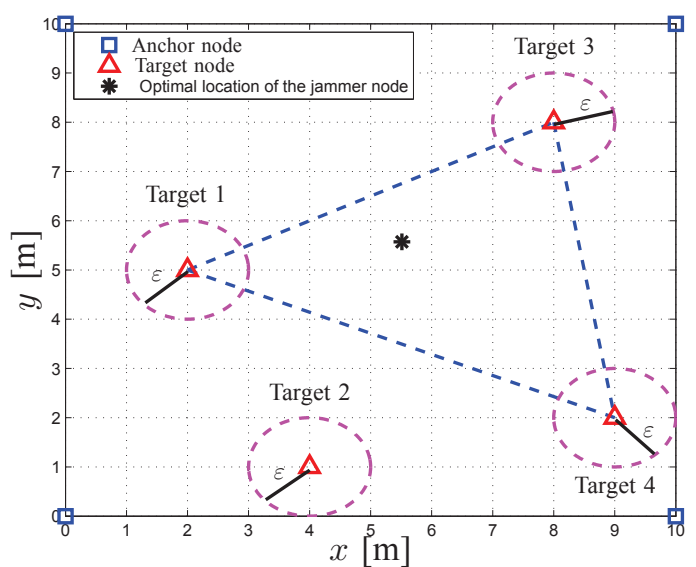

Fig. 2. The network consisting of anchor nodes at [0 0$]$, [10 0], [0 10$]$, and $\left[\begin{array}{ll}10 & 10\end{array}\right] \mathrm{m}$. , and target nodes at $[25],\left[\begin{array}{ll}4 & 1\end{array}\right],\left[\begin{array}{l}8 \\ 8\end{array}\right]$, and $\left[\begin{array}{ll}9 & 2\end{array}\right] \mathrm{m}$.

the jammer node are larger than $\varepsilon=1 \mathrm{~m}$. (that is, the constraints in (16) are ineffective), Proposition 4 is also applicable for this scenario. Namely, two of the CRLBs of the target nodes (target nodes 1 and 3 ) are equalized by the optimal solution, and the optimal location of the jammer node corresponds to the minimum of the max-min CRLBs for all possible subnetworks with three target nodes, which is achieved by any subnetwork with three target nodes that contains target node 1 and target node 3. (In each of these subnetworks, the conditions in Proposition 2 are satisfied with $k=1$ and $i=3$, which in turn implies that $z_{1,3}^{\mathrm{opt}}$ and $\mathrm{CRLB}_{1,3}$ are the optimizer and the optimal value of (16), respectively.)

Next, another scenario with four anchor nodes and four target nodes is investigated, where the node locations are as shown in Fig. 2. When Proposition 4 is employed in this scenario, it is observed that the subnetwork consisting of target nodes 1, 3, and 4 achieves the minimum maxmin CRLB among all possible subnetworks with three target nodes, and the corresponding values are obtained as $\mathrm{CRLB}_{1,3,4}=0.7983 \mathrm{~m}^{2}$ and $z_{1,3,4}^{\mathrm{opt}}=[5.51155 .5717] \mathrm{m}$. Since the distances between the target nodes and $z_{1,3,4}^{\mathrm{opt}}$ are larger than $\varepsilon=1 \mathrm{~m}$.; that is, $\boldsymbol{z}_{1,3,4}^{\mathrm{opt}}$ is an element of $\left\{\boldsymbol{z} \mid\left\|\boldsymbol{z}-\boldsymbol{x}_{i}\right\| \geq \boldsymbol{\varepsilon}, i=1,2,3,4\right\}$, the solution of (16) is the same as that of the subnetwork consisting of target nodes 1, 3, and 4 in this scenario. The calculations also show that the CRLBs of target nodes 1, 3, and 4 are equalized in accordance with the statement in Proposition 4, whereas the CRLB for target node 2 is larger than $\mathrm{CRLB}_{1,3,4}$ for the optimal jammer location.

\section{CONCLUding REMARKS}

The problem of optimal jammer placement has been proposed in order to maximize the minimum of the CRLBs for a number of target nodes in a wireless localization network. Various theoretical results have been obtained for specifying scenarios in which the jammer node is located as close to a certain target node as possible, or the optimal location of the jammer node is determined by two or three of the target nodes. In addition, explicit expressions for the optimal location of the jammer node have been derived in the presence of two target nodes. Numerical examples have provided an illustration of the theoretical results.

\section{REFERENCES}

[1] R. Zekavat and R. M. Buehrer, Handbook of Position Location: Theory, Practice and Advances. John Wiley \& Sons, 2011.

[2] S. Gezici, "A survey on wireless position estimation," Wireless Personal Communications, vol. 44, no. 3, pp. 263-282, Feb. 2008.

[3] S. Gezici, M. R. Gholami, S. Bayram, and M. Jansson, "Optimal jamming of wireless localization systems," in IEEE International Conference on Communications (ICC) Workshops, June 2015.

[4] S. Sankararaman, et. al., "Optimization schemes for protective jamming," in Proceedings of 13th ACM MobiHoc, June 2012, pp. $65-74$.

[5] A. Shankar, "Optimal jammer placement to interdict wireless network services," M.S. Thesis, Naval Postgraduate School, 2008.

[6] M. K. Simon, J. K. Omura, R. A. Scholtz, and B. K. Levitt, Spread Spectrum Communications. Rockville, MD: Comput. Sci. Press, 1985, vol. 1.

[7] M. Weiss and S. C. Schwartz, "On optimal minimax jamming and detection of radar signals," IEEE Trans. Aeros. Elect. Sys., vol. AES-21, no. 3, pp. 385-393, May 1985.

[8] R. J. McEliece and W. E. Stark, "An information theoretic study of communication in the presence of jamming," in Int. Conf. Commun. (ICC'81), vol. 3, 1981, p. 45.

[9] Y. Qi and H. Kobayashi, "Cramér-Rao lower bound for geolocation in non-line-of-sight environment," in IEEE International Conference on Acoustics, Speech, and Signal Processing (ICASSP), vol. 3, May 2002, pp. III-2473-III-2476.

[10] Y. Shen and M. Z. Win, "Fundamental limits of wideband localizationpart I: A general framework," IEEE Transactions on Information Theory, vol. 56, no. 10, pp. 4956-4980, Oct. 2010.

[11] W. W.-L. Li, Y. Shen, Y. J. Zhang, and M. Z. Win, "Robust power allocation for energy-efficient location-aware networks," IEEE/ACM Trans. Netw., vol. 21, pp. 1918-1930, Dec. 2013.

[12] Y. Shen, W. Dai, and M. Win, "Power optimization for network localization," IEEE/ACM Trans. Netw., vol. 22, no. 4, pp. 1337 1350, Aug. 2014.

[13] T. Wang and G. Leus, "Ranging energy optimization for robust sensor positioning with collaborative anchors," in IEEE International Conference on Acoustics Speech and Signal Processing (ICASSP), Mar. 2010, pp. 2714-2717.

[14] H. V. Poor, An Introduction to Signal Detection and Estimation. New York: Springer-Verlag, 1994.

[15] T. Zhang, A. Molisch, Y. Shen, Q. Zhang, and M. Win, "Joint power and bandwidth allocation in cooperative wireless localization networks," in IEEE Conference on Communications (ICC), June 2014, pp. 2611-2616.

[16] A. Goldsmith, Wireless Communications. Cambridge University Press, 2005. 